\title{
Muon reconstruction performance and detector-design considerations for a Muon Collider
}

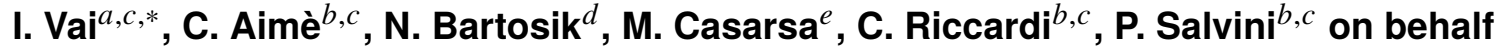 of the Muon Collider Physics and Detectors working group}

${ }^{a}$ Dipartimento di Ingegneria e Scienze Applicate, Università di Bergamo, Viale Marconi 5, Dalmine, Italy

${ }^{b}$ Dipartimento di Fisica, Università di Pavia,

Via Bassi 6, Pavia, Italy

${ }^{c}$ Istituto Nazionale di Fisica Nucleare, Sez. di Pavia, Via Bassi 6, Pavia, Italy

${ }^{d}$ Istituto Nazionale di Fisica Nucleare, Sez. di Torino, Via Giuria 1, Torino, Italy

${ }^{e}$ Istituto Nazionale di Fisica Nucleare, Sez. di Trieste, Via Valerio 2, Trieste, Italy

E-mail: ilaria.vai@unibg.it

A muon collider has a great potential for particle physics giving the possibility to reach the high center-of-mass energy and luminosity of hadron colliders, with a greatly reduced pile up effect. However, a series of challenges arise mainly from the short muon lifetime and the Beam-induced Background. A complete simulation, based on CLIC's ILCSoft software, is ongoing to understand the performance of the full detector. Concerning the muon system, the iron yoke plates are meant to be instrumented with layers of track sensitive chamber to enhance the muon identification. At the moment, according to CLIC geometry, glass Resistive Plate Chambers with readout cells of $30 \times 30 \mathrm{~mm}^{2}$ have been adopted both for the barrel and the endcap region. Other possible solutions, based on MicroPattern Gaseous Detectors, will be discussed considering their characteristics and performance. The results of a preliminary study investigating the muon reconstruction efficiency, Beam-induced Background sensitivity and background mitigation are presented for muon beams collisions at a center-of-mass energy of $1.5 \mathrm{TeV}$.

\footnotetext{
*** The European Physical Society Conference on High Energy Physics (EPS-HEP2021), ***

*** 26-30 July $2021 * * *$

*** Online conference, jointly organized by Universität Hamburg and the research center DESY ***
}

\footnotetext{
${ }^{*}$ Speaker
} 


\section{Introduction}

The muon collider represents an interesting possibility for the future of particle physics: it's a leptonic collider, which means the possibility to perform high precision physics study, with also the advantage that the muon mass is about 200 times heavier than the electron mass, leading to a huge reduction of energy loss through synchrotron radiation. Hence, the possibilty to get a multi-TeV, high luminosity, leptonic collider which would open the door to a large fraction of interesting physics processes [1].

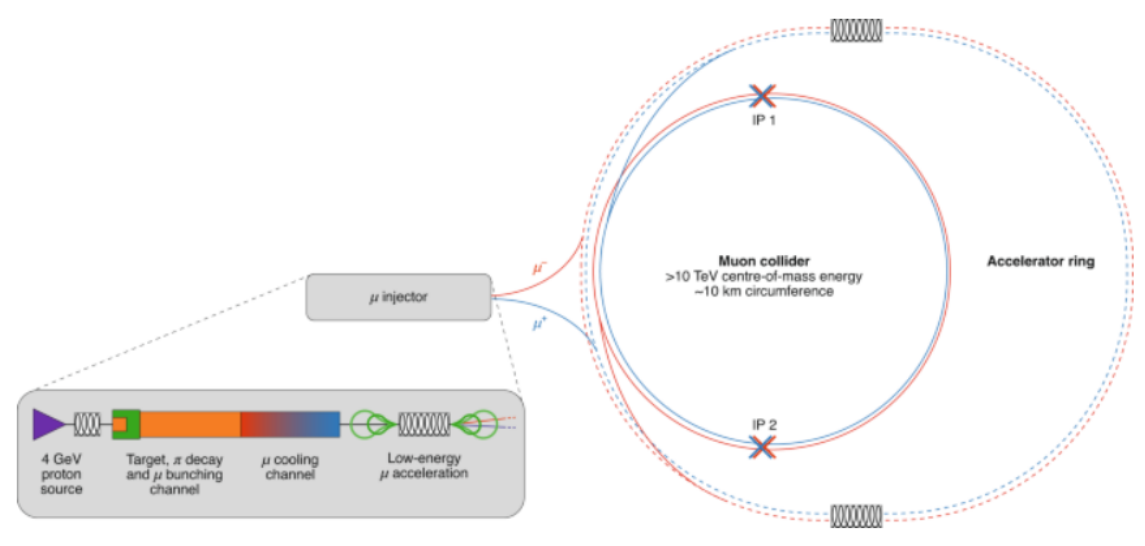

Figure 1: Sketch of a muon collider facility [2]

Fig. 1 shows a sketch of a possibile muon collider facility. The technological challenges related to its realization are not negligible and include in particular the need to produce a large number of muon in small emittance bunches, but also the handling of the so-called Beam Induced Background (BIB). BIB is produced mainly by the decay of muons, which are unstable, during the acceleration process and it represents a huge challenge both for the detector operation and their performance, but also from the safety point of view, because it implies a relevant neutrino hazard [2].

\section{Simulating a muon collider experiment}

A simulation of a full detector installed at a muon collider is then fundamental to understand the feasibility of the project and outline the physics potentials. The current design of a muon collider experiment is based on the CLIC detector [1,3] and shown in Fig. 2. Focusing on the muon system, the iron yoke plates are instrumented with 7 layers of detectors in the barrel (in green) and 6 layers of detectors in the endcaps (in purple); the detector technology currently implemented in simulation is Glass Resistive Plate Chambers (GRPC) [4], with $30 \times 30 \mathrm{~mm}^{2}$ cells. The first perfomance studies show that this configuration allows to reach a very good efficiency in the single muon reconstruction, Fig. 3 left, for particles in the $1 \mathrm{GeV}-1 \mathrm{TeV}$ range, as well as a very good transverse momentum resolution, Fig. 3 right.

However, for the proper evaluation of the performance of the muon system, also the BIB contribution has to be taken in consideration. BIB in the endcap of the muon system is mainly composed by neutrons and photons, with the energy ranges shown in Fig. 4: at a muon collider 


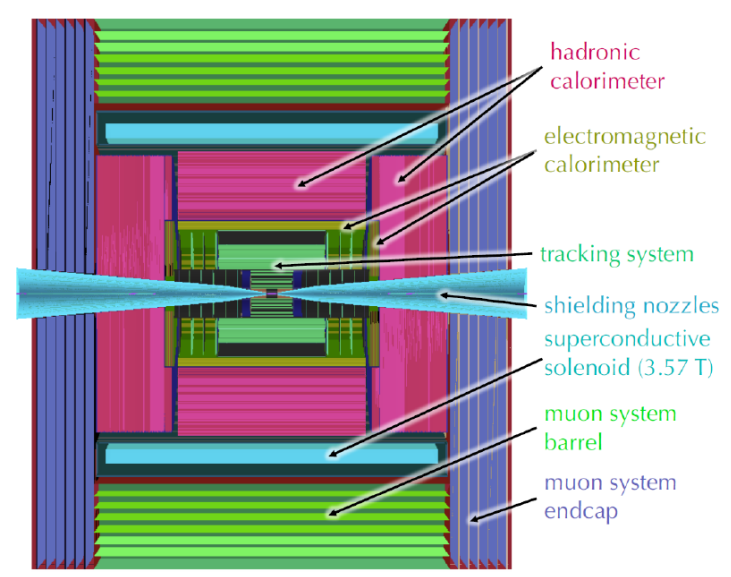

Figure 2: Design of the CLIC detector [3].
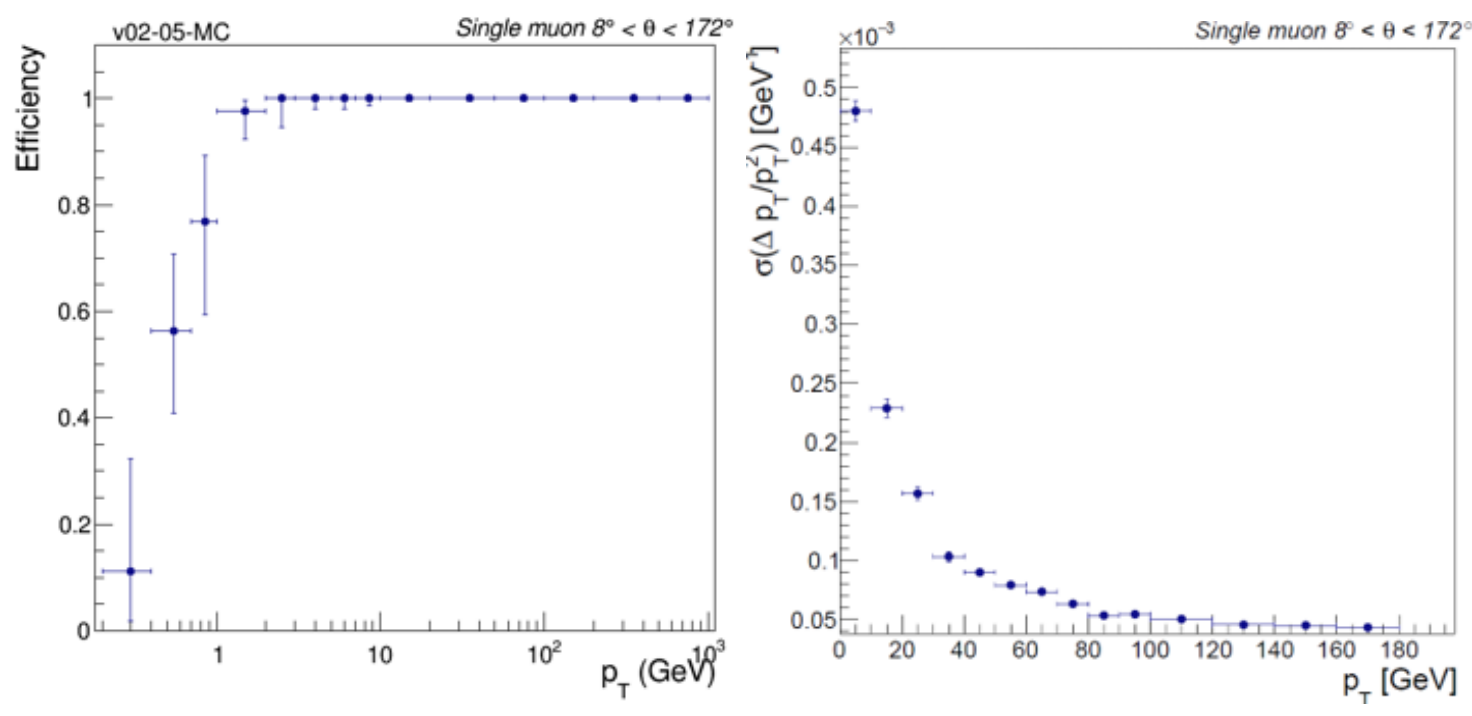

Figure 3: Left: single muon efficiency, defined as the generated particles associated to the cluster, divided by the total generated particles. Right: resolution in the single muon $\mathrm{p}_{T}$ reconstruction: $\Delta \mathrm{p}_{T}$ is the difference between the generated muon $\mathrm{p}_{T}$ and the $\mathrm{p}_{T}$ of the corresponding Pandora [5] reconstructed track.

with $\sqrt{s}=1.5 \mathrm{TeV}$, neutrons have energies up to $2.5 \mathrm{GeV}$, while photons up to $200 \mathrm{MeV}$; the $\mathrm{BIB}$ in the barrel region was not considered in this study as its contibution is orders of magnitude lower than in the endcap. For a more accurate evaluation of the BIB, the endcap was divided in six geometrical regions, based on the $\theta$ angle, shown in different colors in Fig. 4: as expected, the flux in the inner regions, closer to the beam pipe, is almost three order of magnitudes higher than in the outer regions.

The geometrical BIB distribution is confirmed also by the hits distribution shown in Fig. 5 left: they are concentrated around the beam axis in a region small with respect to the whole layer region. From Fig. 5 right is clear however that the current configuration of the muon system would still be able to work properly with these BIB conditions: indeed, a geometrical cut combined for example, 

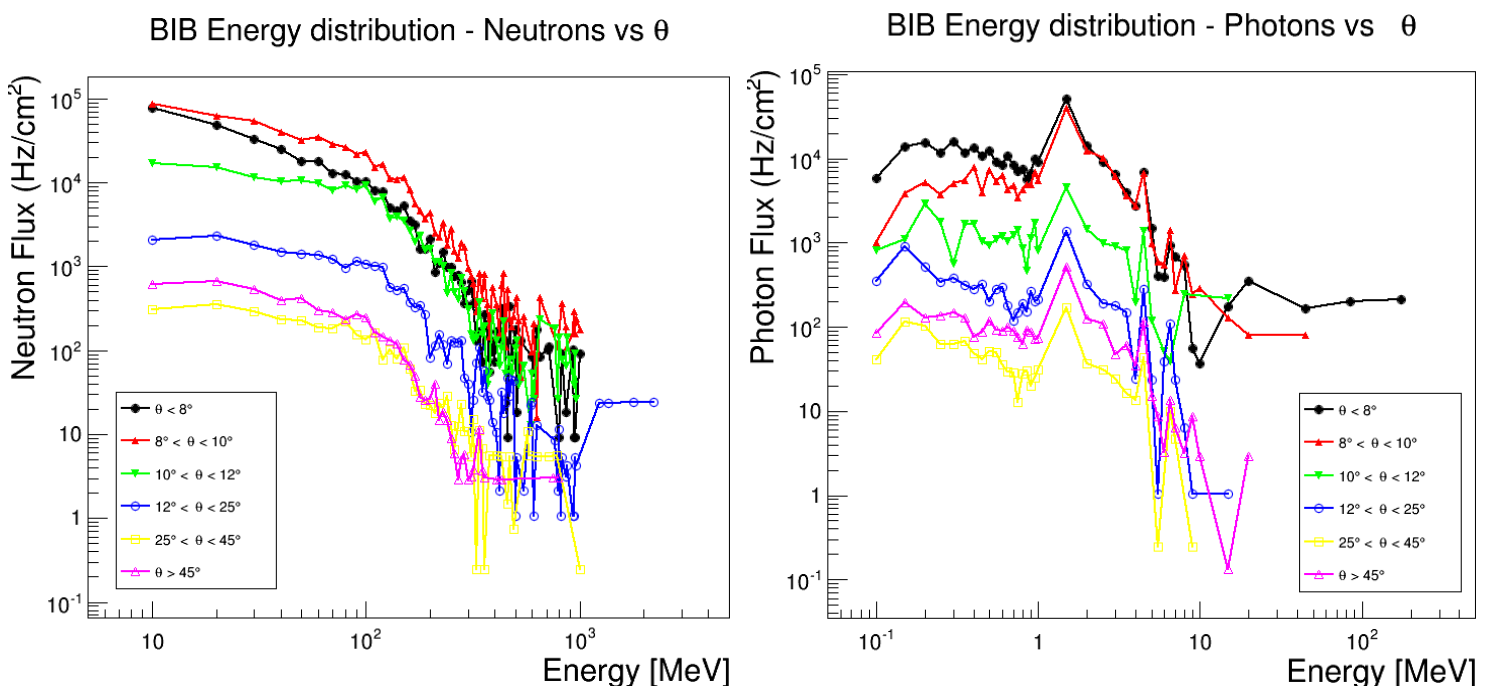

Figure 4: Neutron (left) and photon (right) fluxes from BIB in the endcap of the muon system of a muon collider experiment with $\sqrt{s}=1.5 \mathrm{TeV}$. Different colors represent different geometrical regions of the endcap considered.

with a cut on the track transverse momentum allows to get rid of almost all BIB hits in the muon system.
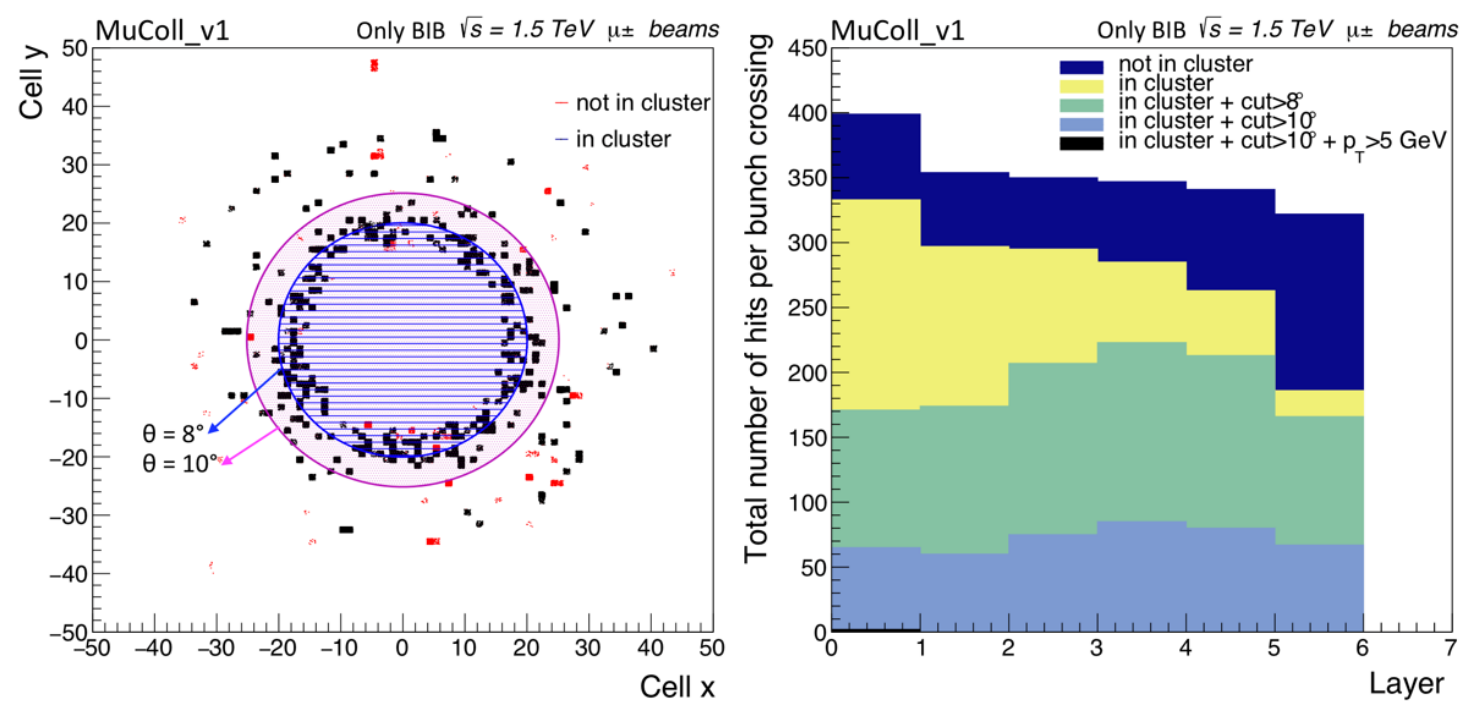

Figure 5: Left: BIB muon hit spatial distribution in the first layer of the muon system endcap. Right: Number of hits per bunch crossing in each layer of the muon system. Different cuts are applied.

\section{Sensitivity to BIB and hit rate estimation in the muon system}

Even if the current BIB conditions do not affect heavily the reconstruction performance in the muon system, a careful study of its effect on the detectors is necessary for the proper choice of the 
technology. Indeed, the rate capability of the detectors has to be taken in consideration, as well as their resistance to radiation. A first step in this direction includes the estimation of the sensitivity of the detectors to BIB and then the evaluation of the hit rate. The sensitivity is the probability for a BIB particle to produce a visibile signal in the detector; it was computed with a standalone Geant4 [6] simulation, where it was defined as

$$
s=\frac{N}{M},
$$

where $N$ is the number of events in which at least one charged particle reaches a sensitive gap, while $M$ is the number of incident particles (counted with a fake layer of air on top of the detector).
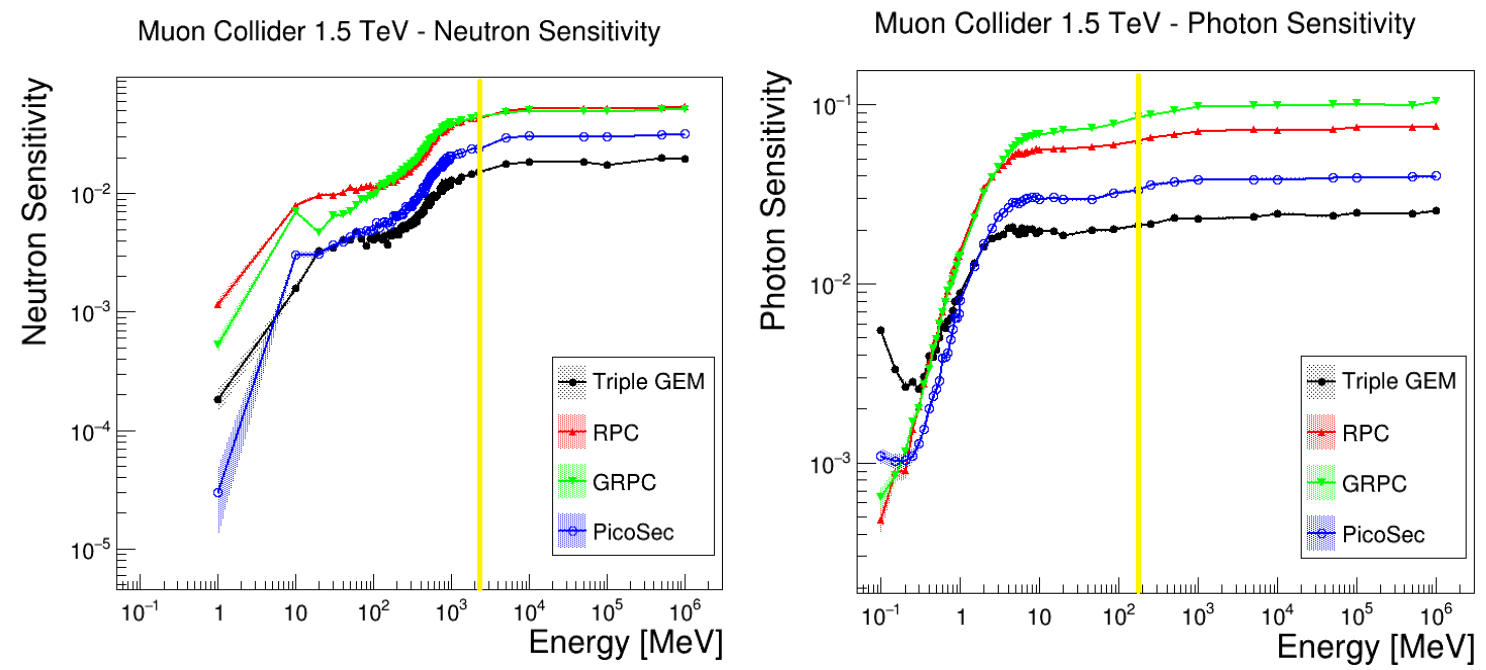

Figure 6: Simulated sensitivity to neutrons (left) and photons (right). Different colors represent different gaseous detector technologies considered.

Fig. 6 shows the computed sensitivity as a function of the energy of the incident particle, for neutrons on the left and photons on the right. Different technologies of gaseous detectors were considered in this study: GRPCs were taken as benchmark, as they are currently implemented in the simulation. They were then compared with classical High Pressure Laminate (HPL) RPC [7], Triple-GEM [8] and a new generation MicroPattern Gaseous Detector (MPGD) with improved time resolution, called Picosec [9]. The results show that sensitivity for MPGDs is in general lower than the one from RPCs, both for neutrons and photons, thanks to the lower material budget of these technologies. The expected hit rate $(H R)$ in the different regions of the endcap is finally computed as

$$
H R=s \times f,
$$

where $s$ is the sensitivity and $f$ is the BIB flux in the considered region and shown in Fig. 7. The hit rate is maximum in the inner regions and decreases moving towards the outer part of the endcap, as expected by the BIB flux behaviour. Moreover, following the differences in sensitivity, the expected hit rate with MPGDs is lower than the one from RPCs. In particular, RPCs and GRPCs in the inner region are at the limit of the standard rate capability. 

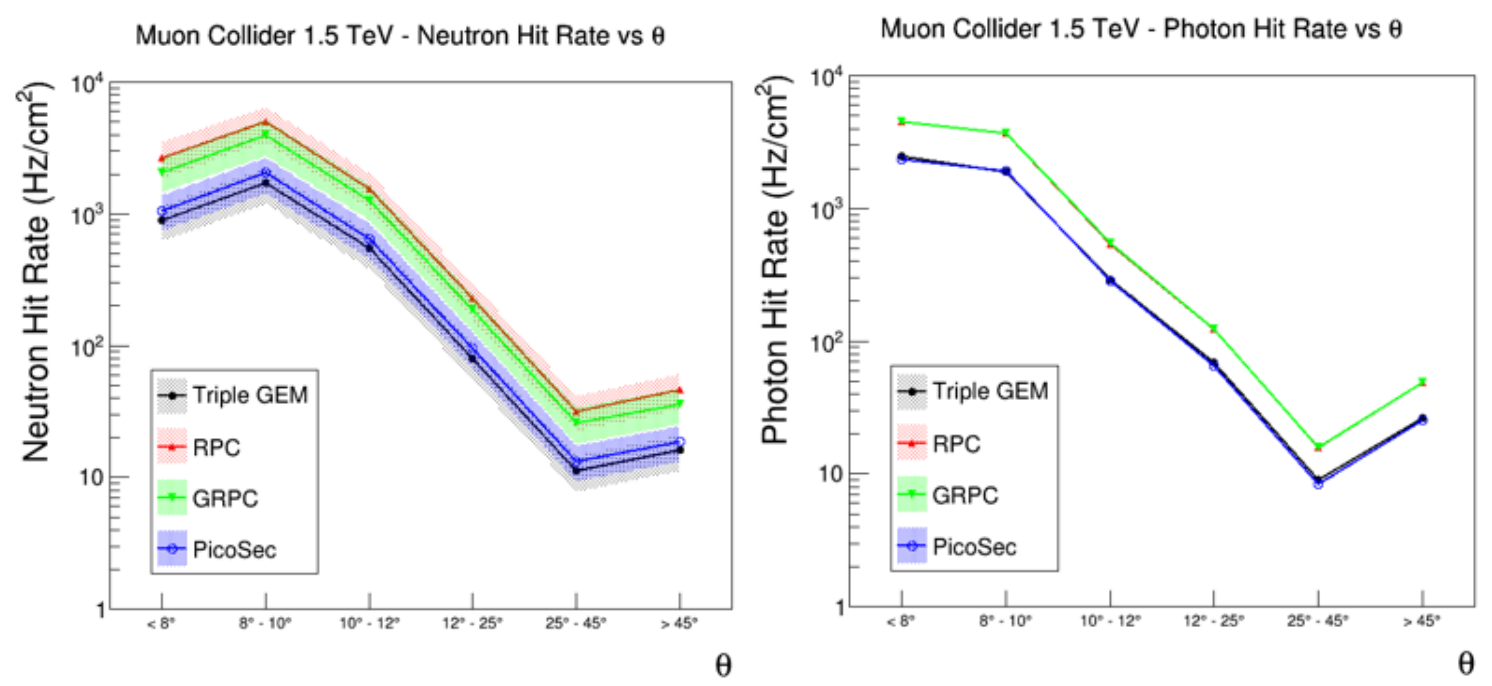

Figure 7: Estimated hit rate from neutrons (left) and photons (right) at $1.5 \mathrm{TeV}$ muon collider. Different colors represent different gaseous detector technologies considered.

\section{Conclusions}

The Muon Collider is a great opportunity for precision physics at high energy and high luminosity. However, its unique environment requires a careful design of the most suitable detectors. In this context, a first study of BIB hit rate in the muon system of a Muon Collider experiment has been performed with a standalone Geant4 simulation. GRPCs, currently implemented in the muon collider simulation, have been compared to Triple GEM, classical RPC and Picosec prototypes. Generally, MPGDs turn out to have lower sensitivities to BIB and then lower expected hit rate. Additional studies will be implemented once the BIB simulation at higher center-of-mass energy will be available. In parallel, analyses focused on the determination of the requested time and space resolutions are ongoing.

\section{Acknowledgments}

This work was performed within the Muon Collider Detector Design and Performance group [10].

\section{References}

[1] N. Bartosik et al., "Detector and Physics Performance at a Muon Collider," 2020 JINST 15 P05001

[2] https://muoncollider.web.cern.ch/

[3] L. Lissen et al., "Physics and Detectors at CLIC: CLIC Conceptual Design Report," arXiv: 1202.5940 [physics.ins-det]

[4] Y. Haddad et al., "High rate resistive plate chamber for LHC detector upgrades," Nucl.Instrum.Meth.A 718 (2013) 424-426 
[5] J. S. Marshall and M. A. Thomson, “The Pandora Particle Flow Algorithm,” arXiv:1308.4537 [physics.ins-det]

[6] J. Allison et al., "Recent developments in Geant4," Nucl.Instrum.Meth.A 835 (2016) 186-225

[7] P. Paolucci et al., "CMS Resistive Plate Chamber overview, from the present system to the upgrade phase I," 2013 JINST 8 P04005

[8] F. Sauli, "The gas electron multiplier (GEM): Operating principles and applications," Nucl.Instrum.Meth.A 805 (2016) 2-24

[9] J. Bortfeldt et al., "PICOSEC: Charged particle timing at sub-25 picosecond precision with a Micromegas based detector," Nucl.Instrum.Meth.A 903 (2018) 317-325

[10] Muon Collider Detector Design and Performance group, https://muoncollider.web. cern.ch/design/muon-collider-detector 